\title{
LA INVESTIGACIÓN CIENTÍFICA EN LA UNIVERSIDAD PERUANA
}

\author{
Manuel Jesús, AMÉZQUITA AMÉSQUITA ${ }^{1}$ \\ Daniel, PATRICIO ÁVILA ${ }^{2}$ \\ Ana Felícita, QUISPE CONDORI ${ }^{3}$ \\ Maritza, SIMÓN GARCÍA ${ }^{4}$
}

\begin{tabular}{|lll|}
\hline Recibido & $:$ & 20.02 .2020 \\
Aceptado & $:$ & 30.06 .2020 \\
Publicado & $:$ & 06.07 .2020 \\
\hline
\end{tabular}

RESUMEN: A cinco años de promulgada la ley universitaria 30220, se realiza una breve revisión al estado actual, en cuanto a presupuesto, producción científica y acciones tomadas en los últimos años, para promover la investigación científica y desarrollo tecnológico del país, por parte del gobierno y las instituciones implicadas, como el Consejo Nacional de Ciencia, Tecnología e Innovación Tecnológica (CONCYTEC), y las universidades peruanas.

Palabras claves: Presupuesto, Investigación científica, Producción científica

\section{Scientific research at the peruvian university}

ABSTRACT: Five years after the enactment of university law 30220, is done a brief review of the current state, in terms of budget, scientific production and actions taken in recent years, to promote scientific research and technological development of the country, by the government and institutions involved, such as the National Council of Science, Technology and Technological Innovation (CONCYTEC), and Peruvian universities.

Keywords: Budget, Scientific research, Scientific production.

\footnotetext{
${ }^{1}$ Universidad Nacional Mayor de San Marcos. Email: xjesmanx@gmail.com

${ }^{2}$ Universidad Nacional Mayor de San Marcos.

${ }^{3}$ Universidad Nacional Mayor de San Marcos. Email: anaquispec03@ gmail.com

${ }^{4}$ Universidad Nacional Mayor de San Marcos.
} 


\section{Journal of the Academy | $33 \mid$}

\section{INTRODUCCIÓN}

En esta nueva etapa de la humanidad, las universidades juegan un papel primordial, ya que estas son las llamadas a generar investigación.

En un principio, las universidades se crearon solo para un determinado grupo de personas, que en cierta forma deberían tener "suerte" para pertenecer a dicha casa de estudios, pues solo la clase dominante podía acceder a ella.

Ante este hecho, la Reforma de Córdoba en el año 1918, Argentina, marcó el primer hito para poder democratizar las universidades, de esta manera, las personas de clase media y baja podían tener el derecho, por primera vez, a ser representadas dentro de las universidades. Es en esta universidad donde el docente es el personaje central, que, gracias a su papel de investigador, ayudará a mejorar las condiciones académicas, generar desarrollo científico, crítico y social en el alumno. Es aquí que, "La investigación y la docencia deben conformar una unidad de acción para el investigador, ya que es esta la mejor manera de aportar al estudiante contenidos que eleven su nivel académico" (Sierra, 2004).

En la actualidad, en el Perú no existe una cultura de investigación, comparada con los países desarrollados. Nos estamos abocando solo a desarrollar lo académico dejando el papel del investigador rezagado. Si bien, "investigar no es una acción privilegiada para sabios y, que al contrario, está al alcance de quien tenga la voluntad de emprender la búsqueda para solucionar problemas, no podemos quedarnos solo en consultas bibliográficas" (Sierra, 2004). Frente a esta situación es importante preguntarnos ¿Será necesaria la investigación en las universidades? A lo que respondemos con un rotundo, sí.

Necesitamos concientizarnos de la necesidad de adquirir métodos y técnicas que nos ayuden a una autentica rigurosidad científica. Hagamos del estudiante un ser crítico, autogestor, generador de desarrollo, capaz de enfrentarse a la vida, resolver problemas con un espíritu de ayuda hacia su semejante.

Ello implicará políticas y estrategias flexibles para viabilizar la investigación en las universidades. Resulta esperanzador entonces, lo manifestado por la presidenta del Consejo Nacional de Ciencia, Tecnología e Innovación Tecnológica (CONCYTEC), Fabiola León-Velarde, al decir que se destinará cerca de 142 millones de soles para financiar formación de recurso humano e investigaciones en Ciencia y Tecnología (Huamaní, 2010). De esta manera instó a docentes e investigadores a concursar por financiamiento de proyectos de investigación para así generar conocimiento científico y generar una mayor institucionalidad para la ciencia en el país.

\section{DESARROLLO}

\section{¿Por qué investigar en el Perú?}

Será importante señalar que, ningún país sale del subdesarrollo sin inversión en investigación científica y Tecnológica. Es impresionante, cómo otros países invierten gran cantidad de su presupuesto, con referencia a su P.B.I., en investigación científica, tal es el caso de Alemania, $2.6 \%$, Francia 2,1\%, Israel 4,6 \%, Suecia 2,7 \%, Finlandia 3,5\%. En América latina, el país con más inversión en investigación es Brasil, con un $1 \%$, mientras que Chile el 0,38 \% y Perú apenas el 0,12\% del Producto Bruto Interno, uno de los indicadores más bajos de América latina y del mundo (Maguiña, 2011). Es por eso que hoy no existen políticas que estimulen la investigación, por el bajo presupuesto que el gobierno de turno viene dando al factor investigación, como veremos más adelante.

Se observó la carencia de recursos humanos calificados en Ciencia y tecnología, el obsoleto equipamiento para la investigación, poca tradición y motivación para publicar, mínimo impulso a la innovación y el desarrollo. "El Perú ha experimentado un importante desarrollo económico, sin 


\section{Journal of the Academy | $34 \mid$}

embargo, todavía persisten brechas en distintos sectores de la sociedad que genera injusticias" (Maguiña, 2011).

Cabe señalar que, ahora la CONCYTEC, está haciendo algunos esfuerzos sectoriales para estimular los programas de posgrado en investigación.

Desde 1551, año en que se creó la Universidad Nacional Mayor de San Marcos, hasta el año 1996, solo había 59 universidades, de las cuales 31 eran privadas y 28 públicas. No obstante, y a raíz del Decreto legislativo 882, emitido por el Congreso de la República y orientada a promover la inversión privada en educación, hubo un notable aumento de universidades que en la actualidad suman 143, 92 privadas y 51 públicas. Este incremento solo demostró que ven a las universidades como un negocio, por ello "cuando la educación superior se piensa y ejecuta con fines de lucro, es difícil que la investigación le sea consustancial (Dager, 2017).

Es evidente que, algunas universidades, solo quieren captar alumnado para incrementar sus ganancias, pues realizar investigación en sí, es cara. Podemos decir entonces que la consecuencia de este decreto 882 , es la poca investigación académica en el sistema universitario.

Por ello la actual Ley Universitaria 30220, otorga a la investigación un rol preponderante revirtiendo esta situación, pues una universidad que no investiga traiciona la esencia misma de la Institución.

El 9 de julio de 2014, se aprobó la Ley Universitaria 30220, con el objetivo de priorizar la reforma de la educación superior, declarándose en reorganización el Sistema Nacional de Evaluación y Certificación y Acreditación de la calidad Educativa (SINEACE).

Cabe señalar que, el punto más importante en esta reforma es sin duda la creación de la Superintendencia Nacional de Educación Superior Universitaria (SUNEDU), cuya misión es, regular las condiciones básicas de calidad, impulsando el proceso de licenciamiento institucional y, de manera prioritaria, velar por el desarrollo de la investigación en las universidades.

\section{El presupuesto y la producción científica.}

El presupuesto de la nación, del estado peruano, tiene en uno de sus anexos la asignación denominada: "Investigación científica y desarrollo tecnológico", el cual ha ido sufriendo notables cambios en los últimos diez años, según podemos apreciar en el diagrama 1.

Si bien la universidad puede ejercer la investigación científica de forma independiente, tendría un mayor impacto con el apoyo del estado y la empresa privada (Medina, 2018). Considerando que es vital el apoyo económico del estado, para escalar posiciones en el ranking de producción científica latinoamericana, hemos realizado un análisis de los presupuestos asignados a la investigación científica desde el año 2009.

Sabiendo que, los presupuestos anuales son aprobados el año anterior al de la denominación, podemos comprobar que la aprobación de los presupuestos de los años 2009 al 2011, fueron aprobados durante el gobierno de Alan García Pérez, mientras que los presupuestos de los años 2012 al 2016, fueron aprobados durante el gobierno de Ollanta Humala Tasso, y finalmente, los presupuestos de los años 2017 al 2019 durante el actual gobierno.

Recopilando la información de los presupuestos nacionales aprobados, disponibles en la página web del Ministerio de Economía y Finanzas (MEF), hemos armado el siguiente diagrama. 
Journal of the Academy $\mid 35$ |

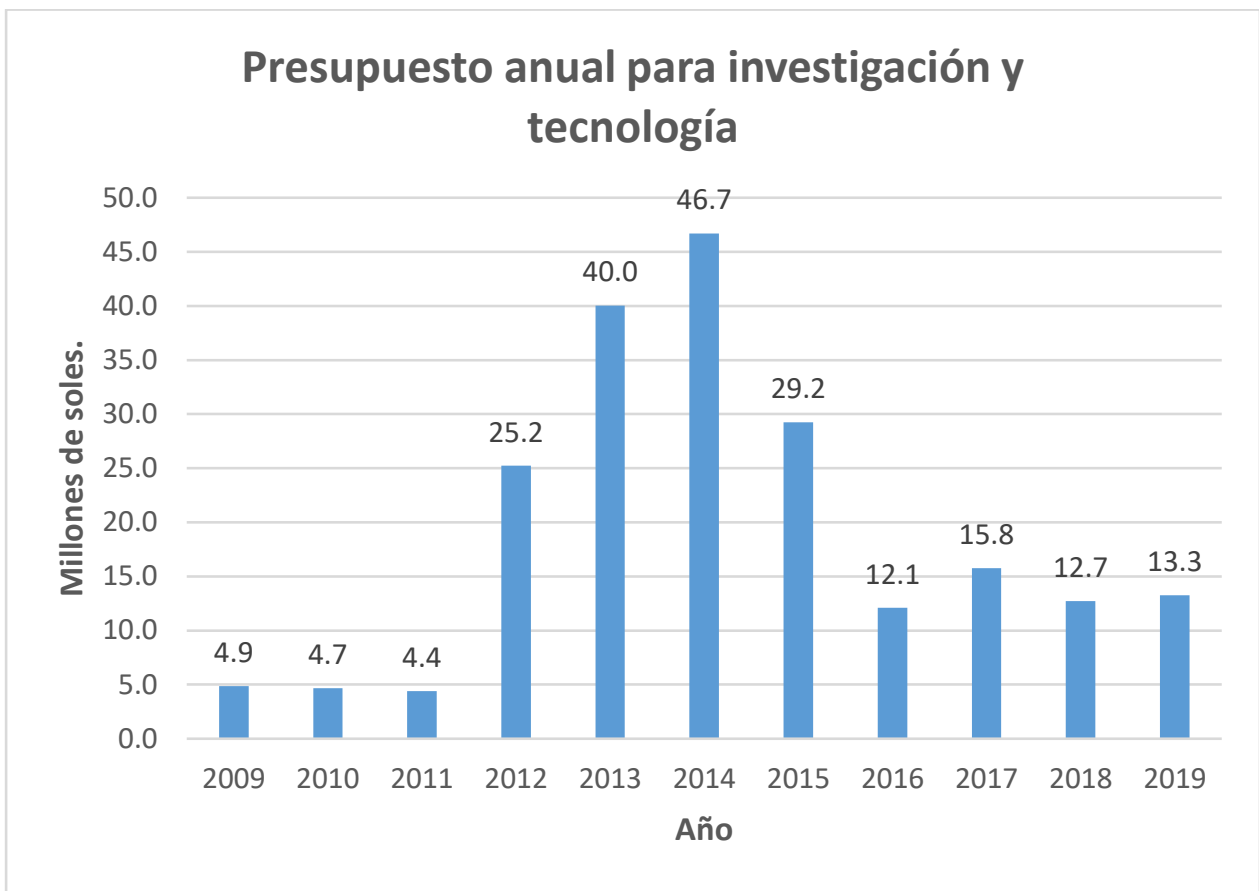

Diagrama 1: Evolución del presupuesto aprobado para la investigación y tecnología.

Fuente: Elaboración propia con información del MEF.

Como ya mencionamos, es notable la diferencia de los presupuestos asignados a la investigación en los diferentes periodos de gobierno, y de la importancia que le dieron. Del diagrama 1 podemos observar que, hemos pasado de menos de 5 millones de soles, en el gobierno de Alan García, a un promedio de 32 millones de soles, durante el gobierno de Ollanta Humala, y finalmente retrocedido, aunque no al nivel del gobierno de Alan García, a un promedio de 14 millones de soles, durante el actual régimen.

Otro dato observado, es que justo después de promulgada la ley universitaria 30220, año 2014, año de la aprobación del presupuesto del año 2015, es que empieza a reducirse el presupuesto para la investigación.

Entendemos que la producción científica está relacionada al presupuesto asignado, pero ¿cómo se evidencia? Si consideramos que, una vez asignado el presupuesto a un proyecto, este tomará un tiempo en arrojar los resultados, digamos dos o tres años, por ejemplo. La producción de artículos se dará luego de esos dos o tres años, y considerando el tiempo de aprobación y publicación de los artículos, es prudente pensar que las publicaciones se evidenciarán en un lapso de entre dos y cuatro años, luego de asignado el presupuesto, que equivale al inicio del proyecto de investigación.

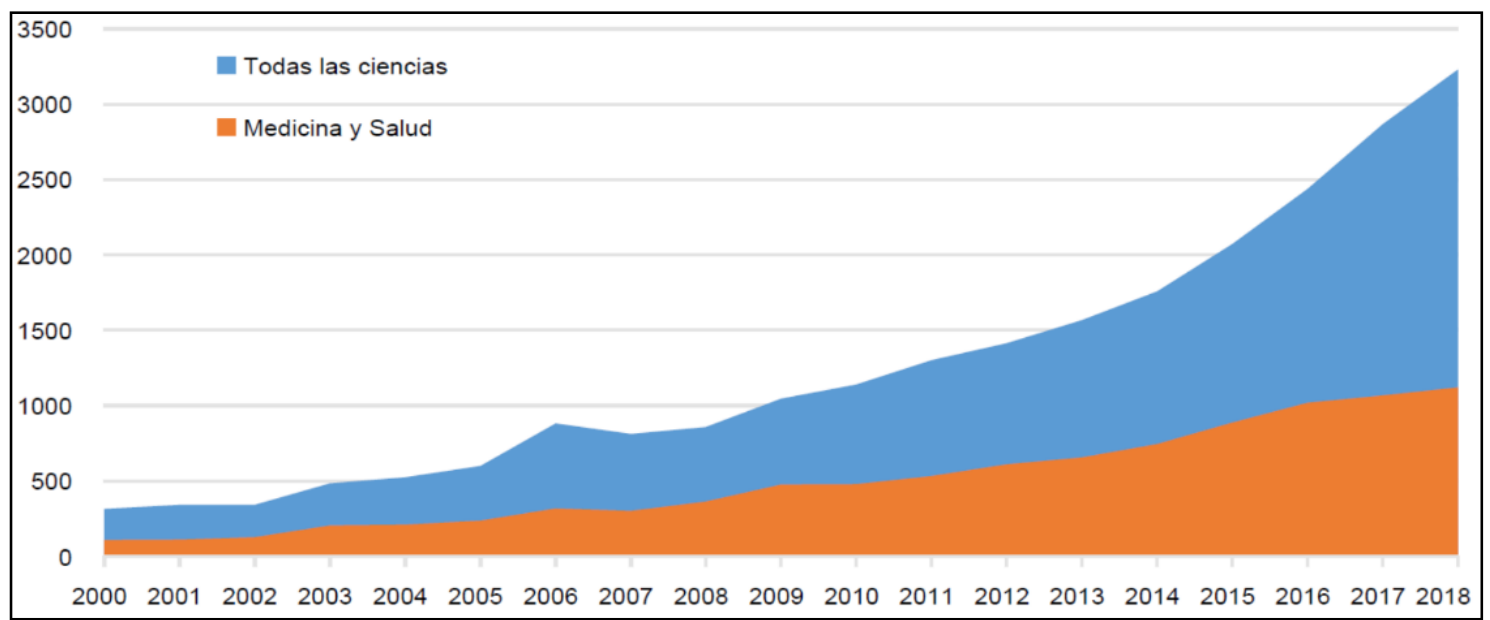

Como citar: Amézquita Amésquita, Manuel Jesús; Patricio Arevalo, Daniel; Quispe Condori, Ana Felícita y Simón García, Maritza (2020). La investigación científica en la universidad peruana. Journal of Academy núm 3, 32-39. www.doi.org/10.47058/joa3.3 


\section{Journal of the Academy $|36|$}

Diagrama 2: Evolución de la producción científica peruana y en el área de medicina y salud en Scopus, 2000-2018 (Moquillaza, 2019)

Fuente: Elaboración propia

Si observamos la producción científica del Perú, expuesta en la gráfica del diagrama 2, podemos apreciar que, a partir del año 2011, el incremento de la producción científica ha ido acelerándose hasta el año 2017, especialmente en el tramo 2014 - 2017, y en el 2018 se desaceleró. Podríamos atribuir este progreso en la producción científica, 2014-2017, a la elevación del presupuesto de los años 2012 al 2014. Así mismo, atribuir la desaceleración en el 2018, a la reducción del presupuesto en el 2015. Lo que nos haría sospechar una desaceleración mayor, en la producción científica nacional, para el presente año y posteriores.

\section{La formación científica.}

En universidades nacionales europeas, norteamericanas y algunas latinoamericanas, los docentes realizan investigación de acuerdo a las asignaturas que enseñan, y acorde a las líneas de investigación de la carrera, produciendo profesionales altamente especializados (Medina, 2018).

En las universidades latinoamericanas, parece haber dos son funciones distintas: docentes formadores y docentes investigadores. No se concibe la idea de que puede existir la docencia sin la investigación. Se supone que docencia e investigación no son actividades separadas, como refiere Bok (1990), el aula debe ser como un laboratorio, con cuestionamientos que impulsen el desarrollo de la investigación, en conjunto, entre docentes y estudiantes, conformándose comunidades académicas (Rojas, 2011), (Medina, 2018).

"Se pueden diferenciar dos tipos de vínculo entre la enseñanza y la investigación, una que parte desde el currículo, llamada investigación formativa y otra vinculada a políticas institucionales, gestionada por lo general por los Vicerrectorados de Investigación, llamada formación para la investigación (Parra, 2004; Moreno, 2005; Guerrero, 2007; Miyahira, 2009)" (Medina, 2018).

La investigación formativa, herramienta del proceso de enseñanza-aprendizaje, haciendo uso del método de investigación, brindado información existente a los estudiantes, sujetos en formación, quienes no son investigadores profesionales (Miyahira, 2009). En cuanto a la formación para la investigación, busca formar profesionales que generen conocimientos, más que solo su uso, claro está, bajo el acompañamiento o liderazgo de un docente (Medina, 2018).

Por lo general, en Latinoamérica, las universidades cumplen más una función profesionalizante, es por ello que algunas universidades, según el área del conocimiento, han optado porque sus estudiantes no se titulen con trabajos de investigación que involucren el método científico, sino por ejemplo con planes de negocio, estudios de mercado, entre otras formas de investigar (Medina, 2018).

Por otro lado, las asignaturas destinadas a dotar de ciertas capacidades investigativas a los estudiantes, son impartidas por docentes con poca experiencia en investigación. Si son docentes a tiempo completo, dejan poco espacio para la investigación, pues la distribución de sus horas, están principalmente orientadas a la docencia o a las actividades administrativas (Parra, 2004).

En el Perú, las principales universidades que tienen una estructura que les permite formar estudiantes investigadores, con reconocimiento internacional por su divulgación científica, son la Pontificia Universidad Católica del Perú (PUCP), la Universidad Peruana Cayetano Heredia (UPCH) y la Universidad Mayor de San Marcos (UNMSM) por mencionar las más destacadas. Las mismas que impulsan la aparición de nuevos investigadores con actividades como: concursos de monografía y tesis con reconocimientos económicos, fondos concursables para el financiamiento de la elaboración de las tesis o de trabajos de investigación entre docentes y estudiantes. Mención aparte para la PUCP que 


\section{Journal of the Academy | $37 \mid$}

cuenta con un programa de apoyo a estudiantes que desde el tercer año de estudios cuentan con recursos y un docente asesor para investigar (Medina, 2018).

\section{Investigación y desarrollo (I+D)}

La expresión investigación y desarrollo (I+D) se refiere a la inversión en investigación en conocimientos científicos y técnicos y al desarrollo de esas tecnologías para obtener nuevos productos, materiales o procesos. Las actividades de innovación y desarrollo, están vinculadas al desarrollo social, generando un impacto mayor cuando existe la relación universidad - empresa estado, llamada también el triángulo de Sábato (Medina, 2018).

Construir una vigorosa infraestructura científica-tecnológica, para asegurar que un país sea capaz de incorporar la ciencia y la técnica a su proceso de desarrollo, es menester de la universidad. Además, transferir a la realidad los resultados de la investigación; acoplar la infraestructura científico-tecnológica a la estructura productiva de la sociedad. (Sábato y Botana, 1968).

"La innovación debe ir unida a la ciencia que produce la universidad y la praxis empresarial, generando el desarrollo económico y tecnológico de las sociedades. (Ramírez \& García, 2010)” (Medina, 2018).

De la triada de Sábato, surgen las llamadas spin offs, start ups, spin outs, centros de transferencia de tecnología, entre otras formas de emprendimiento de individuos, grupos y organizaciones. Un ejemplo de este modelo, en el Perú, es el spin off "Yacu Tec" de la Universidad de Lima, ganador de la edición del 2015 de Start Up Perú, premiado con 50,000 soles, como inversión para el negocio. Este proyecto, no solo busca el bien social, sino también tiene una perspectiva comercial, como todo spin off, empresas formadas desde la investigación en la universidad (Universidad de Lima, 2016).

Lamentablemente en Latinoamérica, los investigadores que pertenecen a la estructura científico tecnológico, suelen vincularse con estructuras fuera de su país de origen, ya que, en estos otros espacios, cuentan con los recursos para investigar, son reconocidos y mejor remunerados. Algunas universidades, que desearon fomentar el tema de investigación y desarrollo, han realizado una serie de campañas para repatriar a sus profesionales, por ejemplo, en el caso peruano, la Universidad Peruana Cayetano Heredia, tiene el programa llamado "Beca de retorno". (Medina, 2018).

Al comparar la situación de Perú en I+D con la del mundo, el resultado es poco alentador. Según el Índice Global de Innovación de 2016, de la Organización Mundial de la Propiedad Intelectual, nuestro país ocupa la posición 71 entre 128 analizados. Este índice compara aspectos integrales relacionados con el desempeño en I+D de las economías, incluyendo el número de investigaciones y el gasto público, así como las instituciones, infraestructura, sofisticación del mercado y el sector empresarial, productos tecnológicos y de conocimiento. Dicho resultado nos ubica por detrás de Chile, México y Colombia, nuestros socios de la Alianza del Pacífico, quienes ocuparon los puestos 40, 61 y 63, respectivamente. (Redacción Gestión, 2017).

Considerando que los Parques Científicos Tecnológicos son una herramienta importante para el vínculo entre la universidad, empresa y estado; el CONCYTEC promueve la creación de estos parques con el fin de aumentar la competitividad del país, mejorar la calidad de vida y el desarrollo de investigación (Medina, 2018)

\section{CONCLUSIONES}

Lamentablemente, como se aprecia, existen grandes esfuerzos por salir de la brecha y apostar más por la investigación en las universidades, esto solo dependerá de la implementación de programas flexibles en pro de la investigación y de mayores recursos que el estado pueda brindar a esta área.

El desarrollo de habilidades investigativas, está ligado a varios aspectos: una formación reflexiva, crítica y valorativa desde la educación básica regular; formación integral exigente y para la 


\section{Journal of the Academy $|38|$}

investigación en el pregrado; requiere también de recursos económicos, asignación de horas, infraestructura, entre otros aspectos.

En el Perú es necesario aumentar los Parques Científicos y Tecnológicos (PCT) que es una de las opciones más interesantes y valiosas para estructurar y articular los sistemas de innovación, tanto en el ámbito nacional como regional. Basados en experiencias en Estados Unidos, Reino Unido y España, se determina que los PCT son exitosos cuando cumplen con cinco factores principales: organización y gestión interna; eficacia en los servicios y actividades y adecuación al entorno; colaboración y liderazgo institucional a nivel local y regional; respaldo político y consenso social; y reputación a nivel nacional e internacional.

Para terminar, nos merece reflexión la siguiente frase, de la Academia Nacional de Ciencias, 1999: "La empresa de la investigación científica, como otras actividades humanas, se construye sobre un cimiento de confianza. Esta confianza perdurará solo si la comunidad científica se dedica a ejemplificar y trasmitir los valores asociados a una conducta científica ética”.

\section{REFERENCIAS BIBLIOGRÁFICAS}

Dager, J. (2017, 12, 11). La investigación en la universidad. El Peruano. Recuperado en 15 de julio de 2019, de https://elperuano.pe/noticia-la-investigación-la-universidad-61958.aspx

Huamaní, Ch, \& Mayta-Tristán, P. (2010). Producción Científica Peruana en medicina y redes de colaboración, Análisis del Citation index 2009. Recuperado en 15 de julio de 2019, de http://scielosp.org/pdf/rpmesp/2010.v27n3/315-325/es

Maguiña, C., \& Galán-Rodas, E. (2011). Situación de la salud en el Perú: la agenda pendiente. Revista Peruana de Medicina Experimental y Salud Publica, 28(3), 569-570. Recuperado en 15 de julio de 2019, de $\quad \underline{\text { http://www.scielo.org.pe/scielo.php?script=sci_arttext\&pid=S1726- }}$ $\underline{46342011000300029 \& \operatorname{lng}=\mathrm{es} \& \operatorname{tln} g=\mathrm{es}}$

Medina, D. (2018). El rol de las universidades peruanas frente a la investigación y el desarrollo tecnológico- Propósitos y Representaciones, 6(2), 703-737. Doi: http://dx.doi.org/10.20511/pyr2018.v6n2.244

Miyahira, J.M. (2009). La investigación formativa y la formación para la investigación en el pregrado. Revista Médica Herediana, 20(3), 119-122.

Moquillaza Alcántara, V., (2019). Producción científica asociada al gasto e inversión en investigación en universidades peruanas. Anales de la Facultad de Medicina, 80(1), 56-59. https://dx.doi.org/10.15381/anales.v80i1.15626

Parra, C. (2004). Apuntes sobre la investigación formativa. Educación y educadores, 7, 57- 77. Recuperado de: http://educacionyeducadores. unisabana.edu.co/index.php /eye/article/view/549.

Ramírez, M., \& García, M. (2010). La alianza Universidad - Empresa - Estado: una estrategia para promover la innovación. Revista EAN, 68, 112. Doi: https://doi.org/10.21158/01208160.n68.2010.500

Redacción Gestión. (2017, 17, 6). Investigación y desarrollo: ¿Dónde nos ubicamos en el mundo?, Diario Gestión, Recuperado de: https://gestion.pe/tecnologia/investigacion-desarrollo-ubicamosmundo-137498

Rojas, C., \& Aguirre, S. (2015). La formación investigativa en la educación superior en América Latina y el Caribe: una aproximación a su estado del arte. Revista Eleuthera, 12, 197-222. https://doi.org/10.17151/ eleu.2015.12.11 


\section{Journal of the Academy $|39|$}

Romaña, T., \& Gros, B. (2003). La profesión del docente universitario del siglo XXI: ¿Cambios superficiales o profundos? Revista de enseñanza universitaria, 21, 7-35. Recuperado de: http://hdl.handle. net/11441/54876

Sierra Pérez, H. (2004). La investigación como prioridad universitaria. Revista Virtual Universidad Católica del Norte, (12).

Sábato, A. (1975). El pensamiento latinoamericano en la problemática ciecnia - tecnología - desarrollo - dependencia. Editorial Paidos - Buenos Aires

Universidad de Lima (23 de marzo del 2016). Primer spin off de la Ulima triunfa en Start Up Perú. Instituto de Investigación Científica. Recuperado de: http://www.ulima.edu.pe/instituto-deinvestigacion-cientifica/noticias/primer-spin-de-la-ulima-triunfa-en-start-peru 